\title{
Malos hábitos de sueño en lactantes: factor de riesgo para síndrome de muerte súbita del lactante. Estudio piloto
}

\author{
Bad sleeping habits in infants: risk factor for sudden infant death syndrome. \\ Pilot study
}

\author{
Trinidad Sánchez ${ }^{\mathrm{a}}$, Dominga Peirano ${ }^{\mathrm{b}}$, Camila Pipino $^{\mathrm{b}}$, Pablo E. Brockmann ${ }^{\mathrm{c}}$
}

aResidente de Enfermedades Respiratorias del Niño. División de Pediatría, Escuela de Medicina, Pontificia Universidad Católica de Chile, Santiago, Chile.

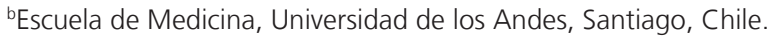

‘Centro del Sueño Pediátrico, Departamento de Cardiología y Enfermedades Respiratorias del Niño, División de Pediatría, Escuela de Medicina, Pontificia Universidad Católica de Chile, Santiago, Chile.

Recibido: 27 de agosto de 2019; Aceptado: 11 de mayo de 2020

¿Qué se sabe del tema que trata este estudio?

La Academia Americana de Pediatría recomienda que los lactantes menores de un año duerman en posición supina para prevenir el síndrome de muerte súbita en lactantes (SMSL).
¿Qué aporta este estudio a lo ya conocido?

En este estudio se describen los hábitos de sueño en una muestra de lactantes chilenos menores de 45 días, siendo frecuente el dormir en posición no segura (de prono o de lado), el colecho y la exposición a tabaquismo pasivo. Educar respecto al dormir seguro es una herramienta efectiva en hacer que los niños duerman en decúbito supino.

\section{Resumen}

La Academia Americana de Pediatría recomienda que los lactantes menores de un año duerman en posición supina para prevenir el síndrome de muerte súbita en lactantes (SMSL). Objetivo: Describir la posición en que duermen un grupo de lactantes y factores de riesgo asociados al SMSL. Sujetos y Método: Estudio piloto, prospectivo concurrente, de lactantes $<45$ días de vida en control sano en Centro Médico San Joaquín UC Christus. Criterios de exclusión: prematurez (edad gestacional < 37 semanas) y patología de base (respiratorias, metabólicas, cardiológicas). Se aplicó encuesta al cuidador principal respecto a datos demográficos y hábitos de sueño, basada en encuesta BISQ (Brief Screening Questionnaire for Infant Sleep Problems) validada en español, dado la inexistencia de instrumentos para < 3 meses. Resultados: Se obtuvo muestra de 100 lactantes de edad 16,78 $\pm 12,88$ días de vida, siendo $57 \%$ mujeres. La madre fue el principal informante (84\%). El 79\% de los lactantes dormían en decúbito supino, $19 \%$ lo hacía de lado y $2 \%$ en prono. El 66\% dormía en cuna en habitación de los padres, $31 \%$ en la cama de los padres. El 74\% se quedaban dormidos durante la alimentación.
Palabras clave:

Sueño;

Lactante;

Síndrome Muerte

Súbita del Lactante; Dormir Seguro

Correspondencia:

Pablo E. Brockmann

pbrockmann@med.puc.cl 
El 28\% de los lactantes estaban expuestos a tabaquismo pasivo. El 91\% cuidadores estaba informado sobre la posición segura de sueño, siendo el principal informante el pediatra (54\%). Conclusiones: En esta muestra se encontró alto porcentaje de lactantes $<45$ días que duermen en posición no segura, siendo frecuente el colecho. Es importante implementar campañas locales de prevención del SMSL que refuercen el hábito de dormir seguro.

\section{Abstract}

The American Academy of Pediatrics recommends, through the implementation of the "Back to Sleep (BTS)" campaign, the supine sleeping position for infant sleeping since it prevents to prevent Sudden Infant Death Syndrome (SIDS). Objective: To describe the sleeping position of a group of infants and the risk factors associated with sudden infant death syndrome (SIDS). Subjects and Method: Prospective pilot study, including infants $<45$ days of life in well-child care visits at a medical center. Exclusion criteria: Preterm-born infant (gestational age $<37$ weeks) and/or comorbidities (pulmonary, metabolic, cardiologic). A brief parental questionnaire was conducted regarding general demographic data and sleep habits. The questioner was based on the BISQ Spanish version, due to the lack of validated instruments for infants < 3-month-old. Results: We included a sample of 100 infants between $16.78 \pm 12.88$ days old ( $57 \%$ girls). Mothers were the main information source (84\%). $79 \%$ of the infants slept in supine position, $19 \%$ slept on their sides, and $2 \%$ in prone position. Regarding the place where the infants slept, $66 \%$ did in their crib in the parents' room and $31 \%$ slept in parents' bed. $74 \%$ of infants fell asleep while being fed. $28 \%$ of infants were exposed to passive smoking at home. $91 \%$ of parents were informed about safe sleep positions, reporting that pediatricians were the main source of information (54\%). Conclusion: We found a high percentage of infants $<45$ days of life who slept in an unsafe position, and frequently co-sleep with their parents. Thus, it is important to implement local SIDS prevention campaigns to reinforce safe infant sleep.
Keywords:

Sleep;

Infants;

Sudden Infant Death

Syndrome;

Sleep Safe

\section{Introducción}

La posición en la que debe dormir un recién nacido y lactante es un tema controversial que ha sido discutido y analizado desde hace muchos años en pediatría, ya que se ha asociado a múltiples patologías ${ }^{1-4}$. El síndrome de muerte súbita del lactante (SMSL) se define como "el fallecimiento de un niño menor de un año, con historia, examen físico y autopsia normales, en el cual no se ha podido establecer una causa definitiva de muerte"'. Se han descrito diversos factores de riesgo, entre los cuales destaca la edad del lactante -siendo mayor el riesgo en menores de 4 meses- y la prematurez $^{3,4}$. Es de mayor riesgo la exposición a tabaquismo prenatal e intradomiciliario, el lugar en el que duerme el niño (mayor si es en la cama de los padres) y el antecedente de un hermano/a fallecido por SMSL, que aumenta el riesgo entre 2 a 10 veces $^{2,5}$. Alrededor del 90\% de las muertes por SMSL ocurre durante los primeros 6 meses de vida, y casi dos tercios de los casos ocurre en la noche, la mayoría de ellos en invierno, y es más frecuente en hombres que en mujeres ${ }^{2}$. Otros factores de riesgo de SMSL son la raza (principalmente nativos americanos y afroamericanos), el consumo de alcohol y drogas ilícitas (ej. heroína) durante el embarazo, la presencia de elementos blandos en la cuna y el sobreabrigo ${ }^{3}$.
En 1985 se observó que en Hong Kong era muy común el hábito de poner a los niños a dormir en posición supina y el SMSL como patología era muy infrecuente. Por el contrario, en Estados Unidos la incidencia de esta patología era mucho mayor, siendo la mayoría de los lactantes puestos a dormir en posición prona. En el año 1992, la Academia Americana de Pediatría (AAP) recomendó cualquier posición excepto la prona para dormir seguro ${ }^{6}$. Luego, en 1994 se implementaron las campañas "Back to Sleep (BTS)" para tomar conciencia de que la posición supina tenía el mayor efecto en prevenir el SMSL ${ }^{6}$ por lo que es la única posición recomendada por la AAP. Al finalizar estas campañas, el rol de los profesionales de salud se volvió crucial. En una revisión sistemática publicada el año $2016^{6}$, respecto a la efectividad de las campañas BTS en los últimos 20 años, se observó que los profesionales de salud seguían siendo los principales promotores de la posición supina como la más segura para el lactante. Esta misma revisión describió una disminución de las tasas de SMSL posterior al inicio de la campaña BTS entre los años 1991 y 1992, en países como Australia de 2,49 a 0,20 por 1.000 recién nacidos vivos (RNV) (1987-2013), USA de 1,37 a 0,87 por 1.000 RNV (1987-2013) y Reino Unido de 2,40 a 0,24 por 1000 RNV (1987-2012) $)^{6}$.

En los últimos 30 años se ha educado a profesionales de salud y padres respecto a la importancia de 
que los lactantes menores de un año duerman en posición supina. Posterior a las campañas BTS, aumentó en un $10 \%$ la toma de conciencia por parte de los profesionales de salud respecto a la posición supina ${ }^{7,8}$. No obstante, el porcentaje de toma de conciencia de padres que deberían evitar poner a sus hijos a dormir en decúbito prono o lateral ha disminuido con el paso del tiempo, de un $97 \%$ a un $90 \%$ hacia finales del año $2000^{7-9}$.

En Chile hasta la fecha actual, no existen estudios conocidos sobre la posición más frecuente en que duermen los recién nacidos y lactantes, ni del grado de información a los padres por parte de profesionales y no profesionales respecto al dormir seguro. El objetivo de este estudio fue describir la posición más frecuente en la que duermen un grupo de lactantes sanos y la presencia de factores de riesgo de SMSL. Además, buscar una eventual asociación entre grado de educación de los cuidadores sobre sueño seguro y posición del niño al dormir.

\section{Sujetos y Método}

\section{Diseño del estudio y participantes}

Se realizó un estudio de tipo piloto, prospectivo concurrente. Se estimó por conveniencia reclutar 100 sujetos, dado que no existen estudios de prevalencia de la posición del lactante al dormir en nuestra población. Se reclutaron recién nacidos de término (edad gestacional mayor de 37 semanas) sanos, menores de 45 días de vida, que acudieron a su control sano del mes en el Centro Médico San Joaquín de la Red de Salud UC Christus. Los criterios de exclusión fueron: prematurez (edad gestacional menor a 37 semanas) y patología de base (respiratorias, metabólicas, cardiológicas, etc.).

Se aplicó una encuesta elaborada por los propios investigadores, basada en las preguntas pertenecientes a la encuesta BISQ (Brief Screening Questionnaire for Infant Sleep Problems $)^{10}$. Esta encuesta evalúa las siguientes variables en lactantes de 6 a 29 meses: lugar donde duerme el niño (cuna en habitación propia, de los padres o hermanos o en la cama de los padres), horas de sueño durante el día y la noche, manera en que se queda dormido (en brazos, solo/a en su cuna, solo/a en presencia del cuidador, mientras se alimenta o acunándolo), posición en la que duerme la mayor parte de la noche (supino, prono o lateral), cantidad de veces que se despierta en la noche y hora de dormir. Se utilizó este cuestionario ya que, según nuestro conocimiento, no se ha desarrollado una encuesta de hábitos de sueño para menores de 3 meses. Sin embargo, BISQ corresponde a una encuesta validada en español ${ }^{11}$ en lactantes de 3 a 30 meses con validez y fiabilidad adecuadas para la evaluación del sueño en lactantes más pequeños que en el trabajo original de Sadeh ${ }^{10}$. Además, se registraron factores demográficos y sociales de los padres, tales como: edad, sexo, nivel educacional de los padres, comuna de residencia y tabaquismo intradomiciliario. Por último, se preguntó respecto a si el cuidador tenía conocimiento sobre la posición más segura al dormir para su hijo, profesional o persona que le había informado (pediatra, enfermera, otro profesional de la salud o familiar cercano) y grado de conocimiento respecto a campañas de prevención de SMSL en Chile. El objetivo de este estudio no fue validar este cuestionario dado que corresponde a uno de tipo piloto, como primera aproximación a los hábitos de sueño en lactantes chilenos.

Se obtuvo la aprobación por el Comité de Ética de la Pontificia Universidad Católica de Chile (proyecto \#170823030). Se solicitó consentimiento informado antes de completar la encuesta, y se entregó a los padres una copia. Se garantizó a los padres la posibilidad de retirarse del estudio en cualquier momento, sin desmedro de futuras atenciones de su hijo(a).

\section{Análisis estadístico}

Para la estadística descriptiva se utilizó media y desviación estándar para las variables continuas y número y porcentaje para las categóricas. Se efectuó un análisis descriptivo, con prueba de Chi-cuadrado para las variables dicotómicas y t de Student para comparar variables cuantitativas. Se realizó una regresión logística de las variables asociadas a la posición de decúbito supino al dormir en lactantes. Se calculó Odds Ratio (OR) con un intervalo de confianza de $95 \%$. Se consideró estadísticamente significativo un valor $\mathrm{p}<0,05$. Todos los análisis fueron realizados mediante el software SPSS Statistics versión 24 para MAC.

\section{Resultados}

Se obtuvo una muestra de 100 recién nacidos de edad 16,78 $\pm 12,87$ días de vida, siendo un $57 \%$ mujeres. La madre fue el principal referente de información $(84 \%)$, y el $86 \%$ de los cuidadores poseía estudios superiores (técnico/universitario) terminados. El 60\% de los niños eran primogénitos. Las características generales de la población se pueden observar en la tabla 1.

Respecto a los hábitos de sueño de los lactantes y factores de riesgo de SMSL (tabla 2), casi el 80\% dormía en decúbito supino y el $20 \%$ en decúbito prono o lateral, destacando una tasa de colecho de $30 \%$. El $73,74 \%$ se quedaban dormidos durante la alimentación. Los cuidadores referían que los niños dormían $8,7 \pm 1,54$ h en la noche y $8,28 \pm 1,98$ h durante el día. El $28,28 \%$ de los lactantes estaban expuestos a taba- 
Tabla 1. Análisis descriptivo de la población estudiada $(n=100)$

\begin{tabular}{ll}
\hline Variables & Resultados \\
\hline Edad en días (media $\pm \mathrm{DE}$ ) & $16,78 \pm 12,87$ \\
Sexo femenino \%, (n) & $57(57)$ \\
Cuidador informante \%, (n) & \\
$\quad$ Madre & $84(84)$ \\
$\quad$ Padre & $15(15)$ \\
$\quad$ Otro & $1(1)$ \\
Nivel educacional cuidador \%, (n) & \\
$\quad$ Medio & $14(14)$ \\
Superior & $86(86)$ \\
\hline
\end{tabular}

Definición de abreviaciones: $\mathrm{DE}$ = desviación estándar

Tabla 2. Hábitos de sueño y factores de riesgo de síndrome de muerte súbita del lactante (SMSL) en el grupo estudiado $(n=100)$

\begin{tabular}{lc}
\hline Variables & Resultados \\
\hline Lugar donde duerme \%, (n) & \\
Cuna en habitación individual & $3(3)$ \\
Cuna en habitación padres & $66(66)$ \\
Cama padres & $31(31)$ \\
Principal forma en la que se queda dormido \%, (n) & \\
Durante alimentación & $73,74(73)$ \\
Acunándolo & $3,03(3)$ \\
En brazos & $13,13(13)$ \\
En su cuna & $5,05(5)$ \\
En su cuna con padres presente & $3,03(3)$ \\
En la cama de los padres & $2,02(2)$ \\
Fumador en el hogar \%, (n) & $28,28(28)$ \\
Fuma dentro del hogar & $3,57(1)$ \\
Fuma fuera del hogar & $96,3(27)$ \\
\hline
\end{tabular}

Definición de abreviaciones: SMSL = Síndrome de muerte súbita del lactante. quismo pasivo; en el 3,57\% de los casos, el cuidador fumaba dentro del hogar.

En relación con la educación en sueño seguro, el 90,9\% estaba informado sobre la posición segura de sueño, siendo el principal informante el pediatra $(54,4 \%)$. Los demás informantes fueron la enfermera en $22,2 \%$, otro profesional de la salud en un $11 \%$ y otra persona no profesional de la salud en un $12,2 \%$. Solo el $16,2 \%$ tenía conocimiento de campañas de prevención de SMSL. El 10,1\% de los cuidadores refería tener un familiar con un hijo fallecido por SMSL.

Al comparar el grupo de recién nacidos que dormía en decúbito supino $(\mathrm{n}=79)$ vs el que dormía en otra posición (prona o lateral) $(\mathrm{n}=21)$ no se encontraron diferencias significativas en edad, nivel educacional de los padres, sexo ni lugar donde dormían (tabla 3). Se encontró que el grupo que no dormía en decúbito supino estaba más expuesto a tabaquismo pasivo (30 vs $27,8 \%, \mathrm{p}=0,04)$ y que sus cuidadores respectivos, tenían menor conocimiento sobre la posición segura al dormir para su hijo/a (76,2 vs 93,7\%, $\mathrm{p}=0,02)$. De los 21 pacientes que no dormían en decúbito supino, 6 dormían en la cama de los padres y de estos, 3 estaban expuestos a tabaquismo pasivo. Los padres de estos últimos 3 niños habían sido educados en posición segura al dormir y 1 tenía un familiar o conocido que había fallecido de SMSL.

Se realizó una regresión logística de las variables asociadas a dormir en decúbito supino ajustando por sexo, nivel educacional del cuidador, lugar donde dormía el recién nacido, información recibida sobre posición segura al dormir, presencia de familiar o caso conocido con SMSL y conocimiento de campañas públicas de prevención de SMSL. El contar con información

Tabla 3. Comparación entre los grupos de lactantes según posición al dormir (decúbito supino vs prono o lateral)

\begin{tabular}{|c|c|c|c|}
\hline Variables & Decúbito supino $(n=79)$ & Decúbito prono o lateral $(n=21)$ & $p$ \\
\hline Edad en días (media $\pm \mathrm{DE}$ ) & $17,35 \pm 13,47$ & $14,61 \pm 10,33$ & 0,39 \\
\hline Sexo femenino \%, (n) & $55,7(44)$ & $61,9(13)$ & 0,26 \\
\hline $\begin{array}{l}\text { Nivel educacional cuidador \%, (n) } \\
\text { Medio } \\
\text { Superior }\end{array}$ & $\begin{array}{l}15,2(12) \\
84,8(67)\end{array}$ & $\begin{array}{c}9,5(2) \\
90,5(19)\end{array}$ & 0,44 \\
\hline $\begin{array}{l}\text { Lugar donde duerme \%, }(\mathrm{n}) \\
\text { Cuna habitación individual } \\
\text { Cuna habitación padres } \\
\text { Cama padres }\end{array}$ & $\begin{array}{l}2,5(2) \\
65,8(52) \\
31,6(25)\end{array}$ & $\begin{array}{c}4,8(1) \\
66,7(14) \\
28,6(6)\end{array}$ & 0,33 \\
\hline $\begin{array}{l}\text { Fumador en el hogar \%, (n) } \\
\text { Fuma dentro del hogar } \\
\text { Fuma fuera del hogar }\end{array}$ & $\begin{aligned} 27,8 & (22) \\
1,3 & (1) \\
26,6 & (21)\end{aligned}$ & $\begin{array}{c}30(6) \\
0(0) \\
28,6(6)\end{array}$ & $0,04 *$ \\
\hline Informado sobre posición segura \%, (n) & $93,7(74)$ & $76,2(16)$ & $0,02 *$ \\
\hline Conocimiento campañas prevención SMSL \%, (n) & $13,9(11)$ & $23,8(5)$ & 0,22 \\
\hline
\end{tabular}

*Se consideró estadísticamente significativo $p<0,05$. Definición de abreviaciones: SMSL = Síndrome de muerte súbita del lactante, DE $=$ desviación estándar. 
respecto al dormir seguro del lactante predecía dormir de espaldas con un OR 9,79 (IC 95\% 1,60 - 59,65, $\mathrm{p}=0,013)$. Otras variables tales como el lugar donde dormía el lactante, sexo, tener un familiar o caso conocido fallecido de SMSL, conocer campañas de prevención de SMSL o el nivel educacional del cuidador principal no tuvieron valor predictivo significativo en este análisis.

\section{Discusión}

Este estudio piloto es una primera aproximación a nivel nacional en describir hábitos de sueño en recién nacidos y lactantes menores de 45 días. Encontramos una alta prevalencia de niños que duermen en posición no segura (20\%), una alta tasa de colecho $(30 \%)$ y un desconocimiento de las recomendaciones actuales de cómo debe dormir un menor de un año para prevenir SMSL.

La posición recomendada al dormir para los menores de un año por la Academia Americana de Pediatría es en decúbito supino ${ }^{12}$. En Argentina, un estudio describió la adherencia a las recomendaciones de sueño en lactantes al mes y a los 4 meses de vida ${ }^{13}$, encontrando una proporción de lactantes que dormían en decúbito supino de un 61,2 y $21,2 \%$, respectivamente. En Brasil el 82,1\% de madres que se encontraban previo al alta del puerperio con su recién nacido manifestaban la creencia de que su hijo/a debía dormir en decúbito lateral o en prono ${ }^{14}$. En Chile, según nuestro conocimiento, este es el primer estudio que describe la frecuencia con que los recién nacidos duermen en decúbito supino. La prevalencia encontrada es similar a lo reportado en otros estudios internacionales ${ }^{15,16}$. Es importante recalcar que la posición decúbito supino al dormir no aumenta el riesgo de aspiración ni asfixia, incluso en niños con reflujo gastroesofágico severo, dado los mecanismos fisiológicos de protección de vía aérea con los que cuentan ${ }^{12}$. Sólo puede tolerarse la posición prona en vigilia, supervisada y controlada, durante periodos postprandiales y como forma de estimulación. Por último, llama la atención que nuestro estudio no encontró una asociación entre mayor nivel educacional del cuidador principal con que el niño duerma en decúbito supino o prono. Esto difiere de lo descrito en otras publicaciones, en las que se describe que, si bien la campaña BTS disminuyó el riesgo absoluto de SMSL en todos los grupos sociales, se observó un aumento de la brecha y la inequidad social, ya que la disminución se vio principalmente en grupos con mayor educación materna y de mayor nivel socioeconómico ${ }^{17}$.

El colecho es un tema controversial. Se define colecho como la situación en que uno o dos adultos llevan a un lactante a dormir en la misma superficie que ellos, ya sea en la cama, sillón, u otra superficie ${ }^{12}$. La prevalencia de colecho es variable en los distintos países, habiendo evidencia a favor del aumento de SMSL y otra no concluyente. En Chile, un estudio internacional respecto a hábitos de sueño en lactantes de 3 meses reveló una prevalencia de colecho de $64 \%$ en una población encuestada de 226 niños $^{18}$, similar a la de países de África y Asia ${ }^{19}$. A pesar de lo frecuente que pareciera ser esta práctica en nuestra población, existen situaciones en que el colecho está contraindicado y ha demostrado ser un riesgo para situaciones de asfixia y de SMSL: en lactantes menores de 4 meses $(\mathrm{OR}=4,7$ a 10,4$)$, presencia de padres fumadores $(\mathrm{OR}=2,3$ a $21,6)$, prematurez, padres con consumo de alcohol o drogas $(\mathrm{OR}=1,66$ a 89,7$)$ y el colecho con persona no progenitor del lactante $(\mathrm{OR}=5,4)^{12}$. En nuestro estudio, todos los lactantes eran menores de 2 meses, encontrándose dentro de la edad de mayor riesgo de SMSL. Nuestro grupo describió previamente que el $81 \%$ de los casos de SMSL registrados en Chile entre 1997 al 2009 ocurrieron antes de los 2 meses de vida ${ }^{2}$. Consideramos que, la tasa encontrada de colecho de $30 \%$, es extremadamente alta dada la edad susceptible de los niños. Cabe destacar que existe un factor étnico y cultural importante asociado a colecho y SMSL. En un estudio británico ${ }^{20}$ en que se encuestaron 2.560 familias de lactantes de 2 a 4 meses se encontró que en las de ascendencia asiática (de Bangladesh, India o Pakistán) había mayor probabilidad de colecho (OR = 8,48 [95\% IC $2,92-24,63]$ ) que en las de raza blanca. Sin embargo, en familias asiáticas había también mayor probabilidad de proteger al lactante de otras situaciones de sueño inseguro tales como: exposición a tabaquismo prenatal, consumo de alcohol en ambos padres, dormir con elementos blandos en la cuna y dormir en un sofá con un progenitor. Esto pudiera explicar la razón por la que, a pesar de haber mayor colecho en poblaciones asiáticas, la prevalencia de SMSL tiende a ser menor ${ }^{6}$.

El tabaquismo de las madres durante el embarazo y de ambos padres en el postnatal es un factor de riesgo para SMSL. El riesgo es acumulativo, teniendo la exposición postnatal a un padre fumador un OR de 2,5 (IC $95 \% 1,2-5,0$ ) y a dos padres, un OR $=5,77$ (IC 95\% $2,2-15,5)^{21}$. Nuestro trabajo describió que casi un 30\% de los niños estaban expuestos a tabaquismo de los padres, siendo significativamente mayor en el grupo que no dormía en posición segura. Esto le entrega un factor de riesgo acumulativo para SMSL, que agregado al colecho, pone considerablemente en riesgo a un lactante en edad vulnerable?.

Finalmente, llama la atención que el grupo que ponía a dormir a su hijo/a en posición no segura tenía un grado significativamente menor de conocimiento sobre las recomendaciones actuales. Posterior al análisis multivariado, el único predictor significativo para que 
el lactante durmiera en decúbito supino fue que el cuidador contara con información respecto de la posición segura al momento de dormir. Esto significa que, con educación precoz respecto a sueño seguro, podría disminuir el porcentaje de niños que duermen en prono o decúbito lateral durante su primer año de vida. Es fundamental centrar los esfuerzos en desarrollar campañas de educación de la población a nivel nacional, ya que nos encontramos ante un problema de salud pública con repercusiones dramáticas en la vida de las familias.

Nuestro trabajo tiene limitaciones. Se realizó un muestreo por conveniencia de un número pequeño de pacientes, siendo no representativo de la población general. Además, se utilizó una encuesta no validada en nuestra población, a pesar de que se encuentra validada en español. Por otro lado, no se pudo profundizar en las razones por las cuales los cuidadores no seguían las recomendaciones de sueño seguro, especialmente la posición para dormir. Por último, dado que el nivel de educación superior de los cuidadores fue bastante más alto respecto a la realidad nacional $(84 \% v s 19,8 \%)^{22}$, podría limitar la extrapolación de los datos.

La principal fortaleza de este estudio piloto es que busca motivar la promoción de hábitos de sueño seguro y el desarrollo de políticas públicas para prevención de SMSL. Se deben realizar proyectos de investigación a nivel nacional que describan los hábitos de sueño de lactantes en todas las regiones de Chile y caractericen otros factores de riesgo modificables ante los cuales puede educarse a la población y prevenir SMSL (ej. sobreabrigo, características de la cuna y uso de elementos blandos en ésta) y factores protectores de SMSL (lactancia materna y uso de chupete). Además, sería interesante investigar las razones por las cuales los cuidadores siguen (o no) las recomendaciones, para de esta manera guiar las estrategias de promoción y prevención de sueño seguro en lactantes.

\section{Conclusiones}

En esta muestra de lactantes se encontró un alto porcentaje de niños que duermen en posición no se- gura, siendo frecuente el colecho. Es importante implementar campañas de prevención de SMSL en Chile que refuercen el dormir seguro para los lactantes. Educar respecto al dormir seguro es una herramienta efectiva en hacer que los niños duerman en decúbito supino.

Los pediatras son los principales educadores sobre dormir seguro en lactantes. Sin embargo, es fundamental educar a profesionales tanto de atención primaria como terciaria acerca de la importancia de la posición de los lactantes durante el sueño, entre otros factores de riesgo de SMSL.

\section{Responsabilidades Éticas}

Protección de personas y animales: Los autores declaran que los procedimientos seguidos se conformaron a las normas éticas del comité de experimentación humana responsable y de acuerdo con la Asociación Médica Mundial y la Declaración de Helsinki.

Confidencialidad de los datos: Los autores declaran que han seguido los protocolos de su centro de trabajo sobre la publicación de datos de pacientes.

Derecho a la privacidad y consentimiento informado: Los autores han obtenido el consentimiento informado de los pacientes y/o sujetos referidos en el artículo. Este documento obra en poder del autor de correspondencia.

\section{Conflicto de intereses}

Los autores declaran no tener conflicto de intereses.

\section{Agradecimientos}

Quisiéramos agradecer a los niños y sus padres que participaron en este estudio, al centro médico que facilitó la entrevista de los padres.

\section{Referencias}

1. Beckwith JB. Defining the sudden infant death syndrome. Arch Pediatr Adolesc Med. 2003;157:286-90.

2. Brockmann PE, Oyarzún MA, Villarroel L, Bertrand P. Síndrome de muerte súbita del lactante: prevalencia y cambios en los últimos años en Chile. Rev Med Chile. 2013;141:589-94.

3. Lister G, Rybin DV, Colton T, et al. Relationship between Sleep Position and
Risk of Extreme Cardiorespiratory Events. J Pediatr. 2012;161:22-25.e1.

4. Adams SM, Ward CE, Garcia KL. Sudden infant death syndrome. Am Fam Physician. 2015;91:778-83.

5. Silvestri JM, Lister G, Corwin MJ, et al. Factors That Influence Use of a Home Cardiorespiratory Monitor for Infants. Arch Pediatr Adolesc Med. 2005;159:1824.

6. de Luca F, Hinde A. Effectiveness of the "Back-to-Sleep" campaigns among healthcare professionals in the past 20 years: a systematic review. BMJ Open. 2016;6:e11435.

7. Rocca Rivarola M, Reyes $\mathrm{P}$, Henson $\mathrm{C}$, et al. Impact of an educational intervention to improve adherence to the recommendations on safe infant sleep. Arch Argent Pediatr. 2016;114:223-31.

8. Hauck FR, Tanabe KO, McMurry T, Moon RY. Evaluation of Bedtime Basics for Babies: A National Crib Distribution Program to Reduce the Risk 
of Sleep-Related Sudden Infant Deaths. J Community Health. 2015;40:457-63.

9. Horne RSC, Hauck FR, Moon RY. Sudden infant death syndrome and advice for safe sleeping. BMJ. 2015;350:h1989.

10. Sadeh A. A brief screening questionnaire for infant sleep problems: validation and findings for an Internet sample. Pediatrics. 2004;113:e570-7.

11. Cassanello P, Díez-Izquierdo A, Gorina N, Matilla-Santander N, Martínez-Sanchez JM, Balaguer A. Adaptation and study of the measurement properties of a sleep questionnaire for infants and pre-school children. An Pediatr. 2018;89:230-7.

12. Moon RY. SIDS and Other Sleep-Related Infant Deaths: Evidence Base for 2016 Updated Recommendations for a Safe Infant Sleeping Environment. Pediatrics. 2016;138:e20162940.

13. Rocca RM, Bosch FJ, Henson DC, et al. Evaluación de la adherencia a las recomendaciones para disminuir el riesgo de Síndrome de Muerte Súbita del lactante. Rev Chil pediatría. 2014;85:4629.

14. Cesar JA, Marmitt LP, Carpena MX, et al. Maternal Knowledge and Unsafe Baby Sleep Position: A Cross-Sectional Survey in Southern Brazil. Matern Child Health J. 2019;23:183-90.

15. Keene Woods N, Ahlers-Schmidt CR, Wipperman J, Williams T. Comparing Self-Reported Infant Safe Sleep From Community - and Health Care - Based Settings. J Prim Care Community Health. 2015;6:205-10.

16. Hirsch HM, Mullins SH, Miller BK, Aitken ME. Paternal perception of infant sleep risks and safety. Inj Epidemiol. 2018;5:23-9.

17. Pickett KE, Luo Y, Lauderdale DS. Widening social inequalities in risk for sudden infant death syndrome. Am J Public Health. 2005;95:1976-81.

18. Nelson EAS, Taylor BJ, Jenik A, et al.
International Child Care Practices Study: infant sleeping environment. Early Hum Dev. 2001;62:43-55.

19. Mileva-Seitz VR, Bakermans-Kranenburg MJ, Battaini C, Luijk MPCM. Parentchild bed-sharing: The good, the bad, and the burden of evidence. Sleep Med Rev. 2017;32:4-27.

20. Ball HL, Moya E, Fairley L, Westman J, Oddie S, Wright J. Infant care practices related to sudden infant death syndrome in South Asian and White British families in the UK. Paediatr Perinat Epidemiol. 2012;26:3-12.

21. Liebrechts-Akkerman G, Lao O, Liu F, et al. Postnatal parental smoking: An important risk factor for SIDS. Eur J Pediatr. 2011;170:1281-91.

22. Ministerio de Desarrollo Social, Gobierno de Chile, Encuesta CASEN 2017. Disponible en http://observatorio. ministeriodesarrollosocial.gob.cl/casenmultidimensional/casen/casen_2017.php 